ARTICLE

\title{
Aerosol influence on energy balance of the middle atmosphere of Jupiter
}

Xi Zhang ${ }^{1}$, Robert A. West ${ }^{2}$, Patrick G.J. Irwin ${ }^{3}$, Conor A. Nixon ${ }^{4} \&$ Yuk L. Yung ${ }^{5}$

Aerosols are ubiquitous in planetary atmospheres in the Solar System. However, radiative forcing on Jupiter has traditionally been attributed to solar heating and infrared cooling of gaseous constituents only, while the significance of aerosol radiative effects has been a long-standing controversy. Here we show, based on observations from the NASA spacecraft Voyager and Cassini, that gases alone cannot maintain the global energy balance in the middle atmosphere of Jupiter. Instead, a thick aerosol layer consisting of fluffy, fractal aggregate particles produced by photochemistry and auroral chemistry dominates the stratospheric radiative heating at middle and high latitudes, exceeding the local gas heating rate by a factor of 5-10. On a global average, aerosol heating is comparable to the gas contribution and aerosol cooling is more important than previously thought. We argue that fractal aggregate particles may also have a significant role in controlling the atmospheric radiative energy balance on other planets, as on Jupiter.

\footnotetext{
${ }^{1}$ Department of Earth and Planetary Sciences, University of California Santa Cruz, Santa Cruz, California 95064, USA. ${ }^{2}$ Jet Propulsion Laboratory, California Institute of Technology, 4800 Oak Grove Drive, Pasadena, California 91109, USA. ${ }^{3}$ Atmospheric, Oceanic and Planetary Physics, University of Oxford, Clarendon Laboratory, Parks Road, Oxford OX1 3PU, UK. ${ }^{4}$ NASA Goddard Space Flight Center, Greenbelt, Maryland 20771, USA. ${ }^{5}$ Division of Geological and Planetary Sciences, California Institute of Technology, Pasadena, California 91125, USA. Correspondence and requests for materials should be addressed to X.Z. (email: xiz@ucsc.edu).
} 
A $s$ on Earth, Jupiter's atmospheric temperature profile exhibits a strong inversion above the tropopause ${ }^{1}$, implying that its middle atmosphere, or the 'stratosphere', is convectively inhibited. Therefore, the energy budget should be dominated by radiation and the stratified middle atmosphere is in global radiative equilibrium. A firstorder question is: which constituents in the atmosphere control this energy balance? About half of the incoming solar radiation on Jupiter penetrates deep into the troposphere and one third is reflected back to space (Fig. 1$)^{2}$. The bulk constituents, hydrogen and helium, are not radiatively active except via $\mathrm{H}_{2}-\mathrm{H}_{2}$ and $\mathrm{H}_{2}-\mathrm{He}$ collisional-induced absorption (CIA) at pressures $>10 \mathrm{hPa}$ (refs 3,4). The next most abundant gas, methane $\left(\mathrm{CH}_{4}\right)$, diffuses upward from the deep atmosphere and heats the stratosphere by absorbing the near-infrared solar flux ${ }^{3-8}$. The methane photochemical products acetylene $\left(\mathrm{C}_{2} \mathrm{H}_{2}\right)$ and ethane $\left(\mathrm{C}_{2} \mathrm{H}_{6}\right)$, together with $\mathrm{H}_{2}-\mathrm{H}_{2}$ and $\mathrm{H}_{2}-\mathrm{He}$ CIA, absorb the upward mid-infrared radiation from the troposphere and re-radiate it to space, resulting in an efficient net cooling of the middle atmosphere ${ }^{3-9}$ to compensate the solar heating.

The global maps of temperature and $\mathrm{C}_{2}$ hydrocarbons were recently retrieved from the Jupiter flyby data from Cassini and Voyager-1 spacecraft in 2000 (refs 4,10-12) and 1979 (refs 4,12), respectively. On the basis of a state-of-the-art radiative transfer model (see Methods section), we investigate the global energy balance of Jupiter ${ }^{4}$. Surprisingly, the global average cooling flux by gaseous constituents in the middle atmosphere is estimated to be $\sim 1.4 \mathrm{~W} \mathrm{~m}^{-2}$, about 1.5 times larger than solar flux absorbed by the stratospheric $\mathrm{CH}_{4}\left(\sim 0.9 \mathrm{~W} \mathrm{~m}^{-2}\right.$; Fig. 1). Vertically, the gas solar heating rate is substantially smaller than the gas thermal cooling at pressures $>10 \mathrm{hPa}$ (ref. 4). The energy imbalance consistently revealed by the Voyager and Cassini data is not a seasonal effect because Jupiter has nearly zero obliquity. The Jupiter-Sun distance was different for the two flybys, varying

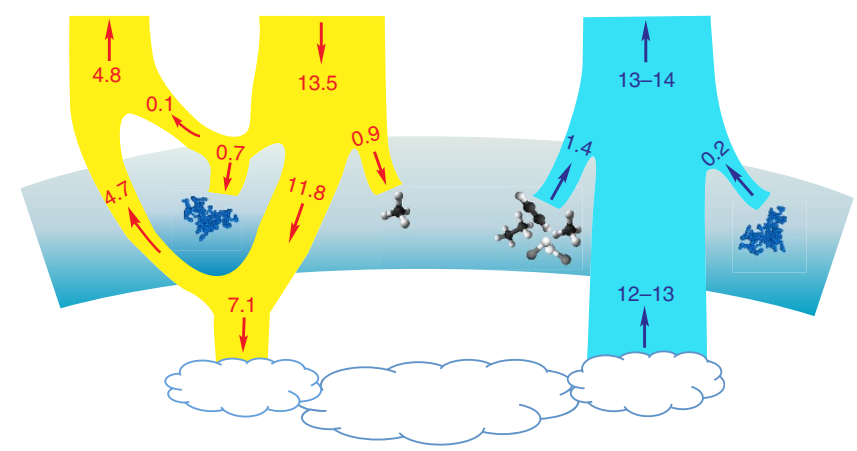

Figure 1 | Globally averaged heating and cooling fluxes on Jupiter. The heating (yellow branch) and cooling (cyan branch) fluxes are in units of $\mathrm{Wm}^{-2}$. The stratosphere is shaded. The heating flux is associated with the incoming solar radiation and the cooling flux is related to the outgoing thermal radiation. Of the $13.5 \mathrm{Wm}^{-2}$ of solar radiation incident to Jupiter's atmosphere, $0.1 \mathrm{Wm}^{-2}$ is reflected back to space and $11.8 \mathrm{~W} \mathrm{~m}^{-2}$ is transmitted to the troposphere. Tropospheric hazes and clouds absorbed $7.1 \mathrm{Wm}^{-2}$ and $4.7 \mathrm{Wm}^{-2}$ is reflected back to space ${ }^{2}$. The remainder of the solar energy is absorbed in the middle atmosphere by fractal haze particles $\left(0.7 \mathrm{Wm}^{-2}\right)$ and $\mathrm{CH}_{4}$ gas molecules $\left(0.9 \mathrm{Wm}^{-2}\right)$. The total outgoing thermal radiation from our radiative calculation is 13-14 W m ${ }^{-2}$, consistent with that from Cassini and Voyager observations ${ }^{9}$. The thermal cooling flux is mainly emitted from the troposphere $\left(12-13 \mathrm{~W} \mathrm{~m}^{-2}\right)$. In the middle atmosphere, the net cooling flux is $1.4 \mathrm{Wm}^{-2}$ emitted by gas molecules $\mathrm{H}_{2}, \mathrm{CH}_{4}, \mathrm{C}_{2} \mathrm{H}_{2}$, and $\mathrm{C}_{2} \mathrm{H}_{6}$ (black and white molecule diagrams). The upper limit of the outgoing thermal flux from the fractal aggregates (blue diagrams) is $\sim 0.2 \mathrm{Wm}^{-2}$ as determined in this study. from northern fall equinox (Voyager) to the northern summer solstice (Cassini), but the global average heating is not altered significantly. Long-term ground-based observations from 1980 to 2000 also show that the global average temperature at $20 \mathrm{hPa}$ does not substantially vary with time ${ }^{10}$, and thus neither does the thermal radiative cooling. The violation of the radiative energy equilibrium thereby suggests the presence of an additional strong heat source other than $\mathrm{CH}_{4}$ in the middle atmosphere of Jupiter, which absorbs the missing $\sim 0.5 \mathrm{~W} \mathrm{~m}^{-2}$.

Here we show that the missing heat source is aerosols, the end product of atmospheric chemistry on Jupiter. As a result of photochemistry, with the help of auroral chemistry, especially at high latitudes (or the 'auroral zone') where high-energy particles penetrate into the atmosphere, complex hydrocarbon compounds can form and eventually coagulate and condense as aerosols, or haze particles ${ }^{13,14}$. On the basis of Cassini imaging science subsystem (ISS) observations ${ }^{15}$, here we derive the globally averaged solar flux absorbed by the stratospheric aerosols of $\sim 0.5$ to $0.7 \mathrm{~W} \mathrm{~m}{ }^{-2}$, more than half of the amount due to $\mathrm{CH}_{4}$ (Fig. 1). The aerosol heating is predominant at middle and high latitudes, exceeding the local gas heating rate by a factor of 5-10. For the first time, we estimate the possible aerosol cooling effect, which might be as important as the cooling via hydrocarbons at high latitudes. We conclude that the aerosols maintain the atmospheric energy balance and must be partially responsible for the stratospheric temperature inversion. That the photochemistry and auroral chemistry control the atmospheric energetics via the production of aerosols suggests that Jupiter exhibits a new regime of atmospheric energy balance that is different from that of the Earth.

\section{Results}

Aerosol heating effect. The global aerosol map has been revealed from images acquired by the ISS during its Jupiter flyby ${ }^{15}$. At low latitudes $\left(40^{\circ} \mathrm{S}\right.$ to $\left.25^{\circ} \mathrm{N}\right)$, an optically thin layer composed of compacted particles with radii $\sim 0.2-0.5 \mu \mathrm{m}$ is found to be concentrated at $\sim 50 \mathrm{hPa}$. The rest of the atmosphere is covered by an optically thick aerosol layer at $10-20 \mathrm{hPa}$ composed of fluffy, fractal particles aggregated from hundreds to thousands of ten-nanometre size monomers, similar to the haze particles on $\operatorname{Titan}^{16,17}$. The fractal dimension of these aggregates is assumed as 2, meaning that their geometric structure of the aggregate particles lies between a long linear chain (fractal dimension of 1) and a fully compacted cluster (fractal dimension of 3). This type of fractal aggregates is consistent with the ISS observations and the polarization observations of Jupiter, whereas spherical particles are not ${ }^{16,17}$. Fractal aggregates are known to be much more absorbing than spherical particles in the ultraviolet and visible wavelengths ${ }^{18}$. For instance, from 0.2 to $1 \mu \mathrm{m}$, an aggregate particle composed of a thousand ten-nanometre monomers can absorb twice as much of the solar flux as an ensemble of $0.07 \mu \mathrm{m}$ individual spherical particles (assumed in ref. 19) with the same extinction optical depth, because the former is less scattering than the latter. Figure $2 \mathrm{~b}$ shows that, at high latitudes, the opacity of the fractal aggregates on Jupiter is considerably larger than the $\mathrm{CH}_{4}$ opacity in the ultraviolet and visible ranges, indicating that these particles can absorb a significant fraction of solar energy at wavelengths where the solar blackbody radiation peaks (Fig. 2a). In addition, long-term observations suggest that the seasonal variation of the Jovian north-south polarization asymmetry is only $\sim 0.5 \%$ (ref. 20 ). The lack of strong temporal variation implies that the fractal aggregates constitute a steady heat source.

The radiative heating calculations demonstrate that the middle atmosphere of Jupiter is heated by two components: aerosols in 

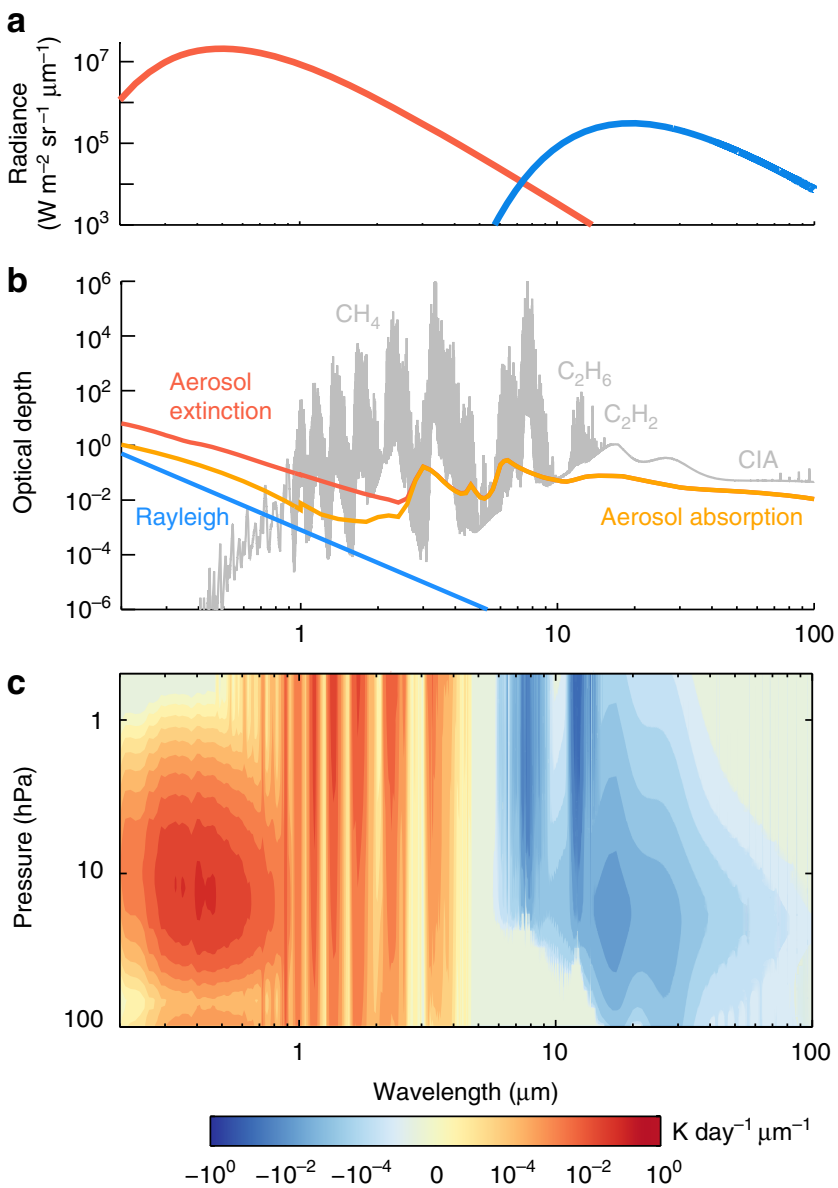

Figure 2 | Spectrally resolved heating and cooling rates and corresponding energy fluxes and opacity. (a) Globally averaged solar radiation received by Jupiter, approximated by a blackbody of 5,778 K (red) and Jupiter thermal radiation in the stratosphere approximated by a blackbody of $150 \mathrm{~K}$ (blue). (b) Total optical depth from the top of the atmosphere to $100 \mathrm{hPa}$ as a function of wavelength at $60^{\circ} \mathrm{S}$. The gas optical depth (grey) includes $\mathrm{H}_{2}-\mathrm{H}_{2}$ and $\mathrm{H}_{2}-\mathrm{He} \mathrm{ClA}$ and $\mathrm{CH}_{4}, \mathrm{C}_{2} \mathrm{H}_{2}$ and $\mathrm{C}_{2} \mathrm{H}_{6}$ absorption. The non-gas components include Rayleigh scattering (blue), fractal aggregate aerosol extinction (red) and the aerosol absorption (orange). (c) Spectrally resolved zonally averaged solar heating $(0.2-5 \mu \mathrm{m})$ and cooling $(5-100 \mu \mathrm{m}) \mathrm{map}$ at $60^{\circ} \mathrm{S}$. Absolute values of the heating/cooling rates that are $<10^{-6} \mathrm{~K}$ per day per $\mu \mathrm{m}$ are not shown. Solar heating dominates shortwards of $5 \mu \mathrm{m}$ while Jupiter thermal cooling (shown in negative values here) dominates longwards of $5 \mu \mathrm{m}$. Contributions from the $\mathrm{H}_{2}-\mathrm{H}_{2}$ and $\mathrm{H}_{2}-\mathrm{He} \mathrm{CIA}$ and gas vibrational-rotational bands are shown. Aerosol heating is important in the ultraviolet and visible regions and aerosol cooling is important in the mid-infrared region beyond $11 \mu \mathrm{m}$.

the ultraviolet and visible wavelengths and $\mathrm{CH}_{4}$ in the near-infrared. Figure $2 \mathrm{c}$ shows the spectrally resolved zonally averaged heating rate as a function of wavelength and pressure at $60^{\circ} \mathrm{S}$. At near-infrared wavelengths longer than $0.9 \mu \mathrm{m}$, strong $\mathrm{CH}_{4}$ bands completely dominate the heating with minor contributions from $\mathrm{H}_{2}-\mathrm{H}_{2}$ and $\mathrm{H}_{2}-\mathrm{He}$ CIA (Fig. 2b). At shorter wavelengths, heating by the fractal aggregates is predominant. The maximum aerosol heating occurs near the wavelength of the solar spectrum peak $(\sim 0.5 \mu \mathrm{m})$, but slightly shortwards owing to the increasing absorption of aerosols towards the ultraviolet. At $60^{\circ} \mathrm{S}$, the integrated aerosol heating rate over all wavelengths can reach $\sim 0.2 \mathrm{~K}$ per day at $10 \mathrm{hPa}$, where it exceeds the $\mathrm{CH}_{4}$ heating rate by a factor of 10 (Fig. 3a). The aerosol heating rate appears to a
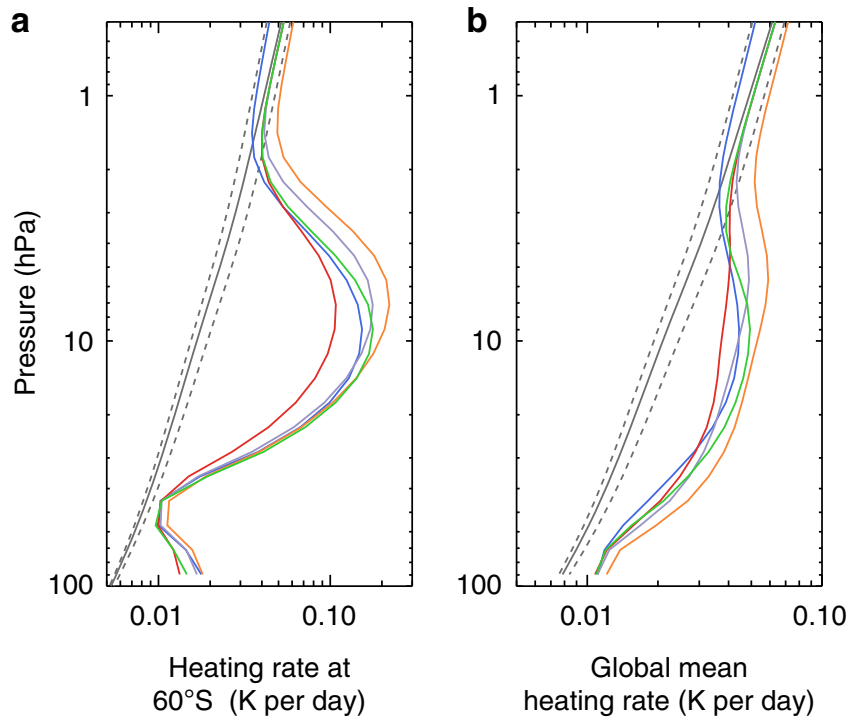

Figure 3 | Vertical heating rate profiles. (a) Zonally averaged heating rates at $60^{\circ} \mathrm{S}$; (b) Globally averaged heating rates. The gas-only calculations are shown in black. The dashed black lines show the possible gas heating rates due to the uncertainty of $\mathrm{CH}_{4}$ profiles. The coloured lines represent different aerosol retrieval solutions. Cases $\mathrm{H} 1-\mathrm{H} 5$ correspond to the green, red, purple, blue and orange curves, respectively. See Table 1 for detailed input information of the cases.

decrease rapidly above $5 \mathrm{hPa}$ and does not contribute significantly to the total heating rate in the upper stratosphere. However, this conclusion is not certain since the resolution of the near-infrared spectra $^{21}$ is not sufficient to fully characterize the upper stratosphere. On the basis of higher-resolution observations ${ }^{22}$, another aerosol layer was found above $5 \mathrm{hPa}$ at the poles that might contribute to the local heating rate in the upper stratosphere, though not to the total energy budget of the middle atmosphere due to its lower density at those levels.

On the basis of Cassini observations, the globally averaged heat flux absorbed by the stratospheric aerosols is $\sim 0.5-0.7 \mathrm{~W} \mathrm{~m}{ }^{-2}$, more than half of the amount due to $\mathrm{CH}_{4}$ (Fig. 1). The maximum globally averaged aerosol heating rate of $\sim 0.03 \mathrm{~K}$ per day occurs at $\sim 10 \mathrm{hPa}$, comparable to the total near-infrared $\mathrm{CH}_{4}$ heating rate at the same pressure level. But the globally averaged heating rate including aerosols is about twice that of the gas-only heating rate at pressures $>20 \mathrm{hPa}$ (Fig. 3b). Spatially, the aerosol heating is predominant at middle and high latitudes, which is attributable to the optically thick fractal aggregates layer (Fig. 4b). Therefore, the aerosol heating naturally, if not coincidentally, compensates for the energy deficit due to gas heating and cooling at the appropriate pressure levels (Fig. 4e).

Owing to the existence of degenerate solutions in the interpretation of ISS observations ${ }^{15}$, we performed sensitivity tests to estimate the uncertainty ranges of the aerosol heating rate. The tropospheric haze and cloud are treated as an effective cloud layer in the troposphere ${ }^{15}$. Our tests show that the effective cloud albedo and phase function within the retrieved uncertainties has insignificant effect on the stratospheric heating rate. The heating rate is more sensitive to the total optical depth and single scattering albedo of the stratospheric aerosols. Given the constraints from the Cassini ISS observations, testing the sensitivity of the heating rate to each individual aerosol parameter is inappropriate. However, multiple solutions still exist in the aerosol retrieval ${ }^{15}$. Therefore, we adopted five typical retrieval solutions for the middle and high latitudes, namely cases 

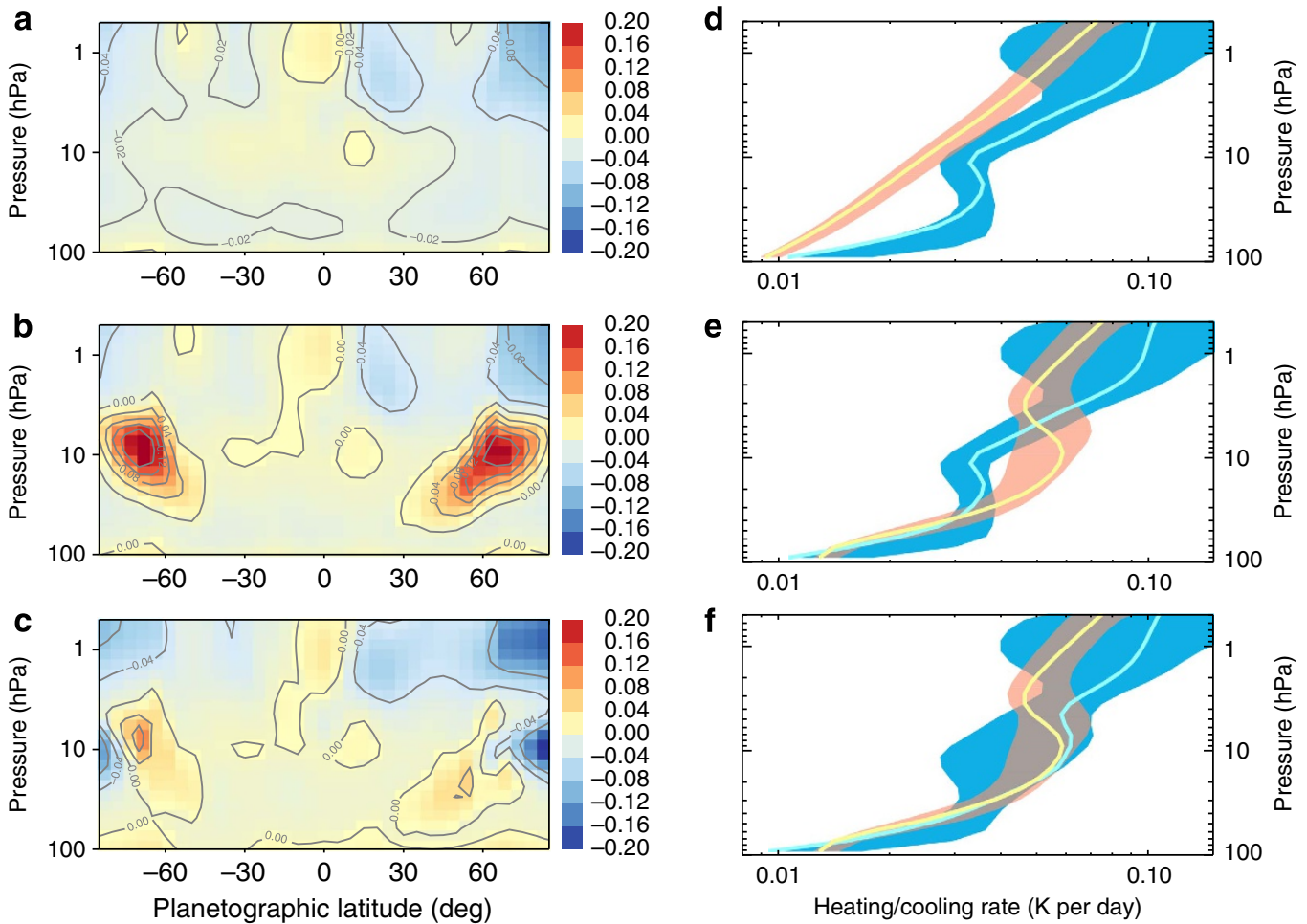

Figure 4 | Radiative balance calculation results in the middle atmosphere of Jupiter based on Cassini flyby observations. (a) Net radiative heating rate map (in units of K per Earth day) without aerosols; (b) net radiative heating rate map with aerosol heating; (c) net radiative heating rate map with aerosol heating and cooling; (d) globally averaged heating (yellow with pink shading) and cooling (cyan with blue shading) profiles without aerosols; (e) globally averaged heating and cooling profiles with aerosol heating; and (f) globally averaged heating and cooling profiles with aerosol heating and cooling. The uncertainty ranges are shaded.

\begin{tabular}{|c|c|c|c|c|}
\hline Case & $\mathrm{CH}_{4}$ mixing ratio & $k$ (UV1) & $k$ (CB3/MT3) & Colour \\
\hline H1 (Nominal) & $1.8 \times 10^{-3}$ & $2 \times 10^{-2}$ & $1 \times 10^{-3}$ & Green \\
\hline $\mathrm{H} 2$ & $1.8 \times 10^{-3}$ & $6 \times 10^{-3}$ & $1 \times 10^{-4}$ & Red \\
\hline $\mathrm{H} 3$ & $1.8 \times 10^{-3}$ & $8 \times 10^{-2}$ & $4 \times 10^{-3}$ & Purple \\
\hline $\mathrm{H} 4$ & $1.5 \times 10^{-3}$ & $2 \times 10^{-2}$ & $1 \times 10^{-3}$ & Blue \\
\hline H5 & $2.5 \times 10^{-3}$ & $2 \times 10^{-2}$ & $1 \times 10^{-3}$ & Orange \\
\hline
\end{tabular}

H1-H5 in Table 1. Those cases were designed to explore the parameter space within the uncertainties of the $\mathrm{CH}_{4}$ mixing ratio $^{4,23}$ and the imaginary part of the refractive indices (hereafter $k$ values) of the UV1 and CB3/MIT3 channels of Cassini ISS ${ }^{15}$. All cases provide good fits to the limb-darkening observations from Cassini ISS (Table 1).

The heating rate calculation at $60^{\circ} \mathrm{S}$ (Fig. 3a) shows that the maximum heating rate including aerosols is about a factor of 2 larger than the minimum at $\sim 10 \mathrm{hPa}$. The maximum (H3) and minimum (H2) occurs when the $k$ value of the UV1 channel reaches the upper and lower bound, respectively, outside which the UV1 limb-darkening profiles cannot be explained ${ }^{15}$. On a global average (Fig. 3b), the maximum heating rate including aerosols is about a factor of 1.5 larger than the minimum (shaded in red in Fig. 4e,f).

Aerosol cooling effect. Aerosols could also cool the middle atmosphere of Jupiter but this effect has not been explored in previous studies. The mid-infrared optical properties of aerosols produced in a hydrogen-dominated environment have not been measured experimentally. But at wavelengths shorter than $2.5 \mu \mathrm{m}$, previous laboratory experiments found that the $k$ values of aerosols produced in a $\mathrm{CH}_{4} / \mathrm{H}_{2}$ gas mixture could be either larger or smaller than their counterpart in the $\mathrm{CH}_{4} / \mathrm{N}_{2}$ mixture, depending on the chemical composition and environmental pressure $^{24,25}$. Therefore, the fractal aggregates on Jupiter might have non-negligible opacity in the mid-infrared compared with $\mathrm{H}_{2}-\mathrm{H}_{2}$ and $\mathrm{H}_{2}-\mathrm{He}$ CIA if their optical constants behave like the aerosols on Titan, which are strongly absorbing with almost no scattering beyond $5 \mu \mathrm{m}$ (Fig. 2b) ${ }^{26,27}$. To estimate the possible cooling effect from aerosols, we included aerosol absorption in a non-linear inversion model to fit the spectra from Cassini CIRS ${ }^{28}$. However, owing to insufficient sensitivity of CIRS observations to the Jovian aerosol opacity, a pure-gas (that is, non-detection of aerosols) model is also able to fit the spectra ${ }^{4,10-12}$. Future analysis on the possible $\mathrm{C}-\mathrm{H}$ bending vibrational features of aerosols at 1,380 and $1,460 \mathrm{~cm}^{-1}$ that have been detected on $\operatorname{Titan}^{27}$ might provide more constraints on the infrared opacity and chemical structure of aerosol particles on Jupiter. Here we aim to derive the upper limit of aerosol opacity from the CIRS spectra and estimate the upper bound of the aerosol thermal infrared cooling.

For each latitude, we included aerosols in the Non-linear optimal Estimator for MultivariatE spectral analySIS (NEMESIS) model $^{28}$ and retrieved the temperature profile and the mixing ratios of $\mathrm{C}_{2} \mathrm{H}_{2}$ and $\mathrm{C}_{2} \mathrm{H}_{6}$ following the procedure detailed in ref. 4. Owing to the lack of Jupiter-analogue aerosol measurements in mid-infrared, we tested several $k$ values based on the laboratory tholin results ${ }^{26}$ and recently derived $k$ values from Cassini observations on $\operatorname{Titan}^{27}$. The latter shows cooling 2-3 times smaller than the former and exhibits different wavelength dependence. We gradually increased the $k$ values 
Table 2 | Sensitivity cases for CIRS retrieval and cooling rate calculation.

\begin{tabular}{|c|c|c|c|c|}
\hline Case & $k$ values in the mid-infrared & $\chi^{2} / \mathrm{N}\left(600-850 \mathrm{~cm}^{-1}\right)$ & $\chi^{2} / N\left(1,225-1,325 \mathrm{~cm}^{-1}\right)$ & Colour \\
\hline $\mathrm{C} 1$ & Pure gas, no aerosol & 0.544 & 1.094 & Red \\
\hline $\mathrm{C} 2$ & Titan tholin experiment ${ }^{26}$ & 0.543 & 1.042 & Blue \\
\hline $\mathrm{C} 3$ & Titan CIRS observations ${ }^{27}$ & 0.541 & 1.090 & Orange \\
\hline C5 & Five times of $\mathrm{C} 2$ values & 0.607 & 1.964 & Brown \\
\hline
\end{tabular}

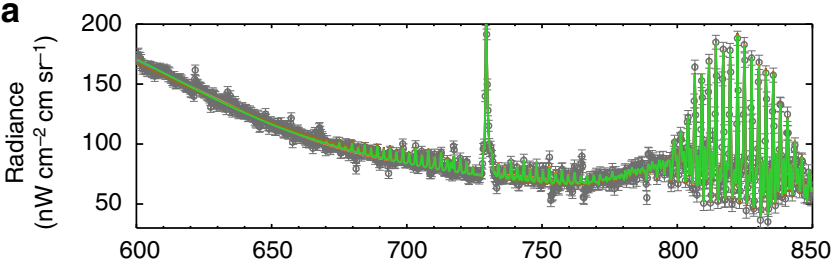

b

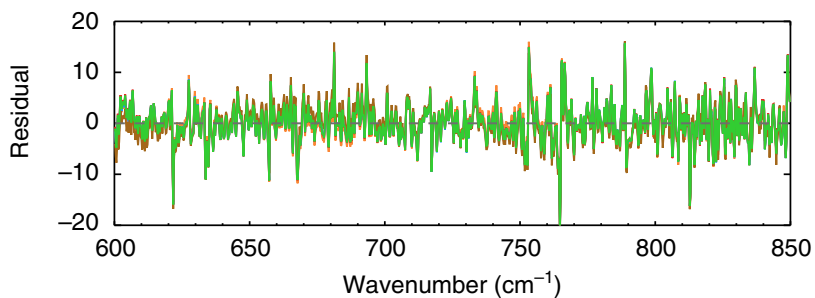

C

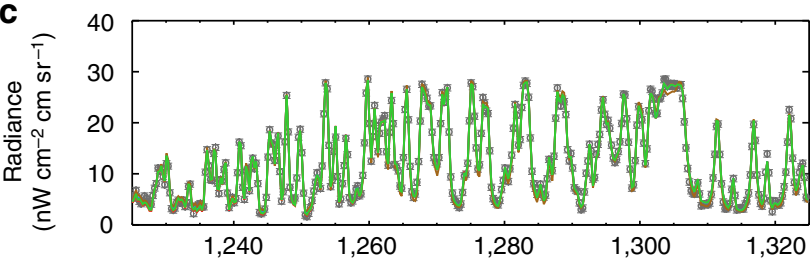

d

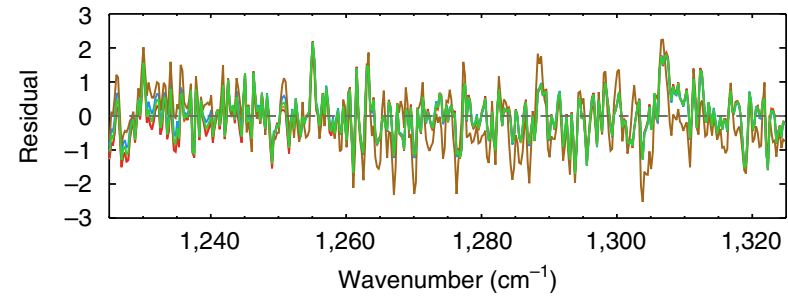

Figure 5 | Spectral inversion results at $\mathbf{5 7 ^ { \circ }} \mathbf{S}$. (a) Spectra at $600-850 \mathrm{~cm}^{-1}$ region $\left(\mathrm{H}_{2}-\mathrm{H}_{2}\right.$ and $\mathrm{H}_{2}-\mathrm{He} \mathrm{ClA}, \mathrm{C}_{2} \mathrm{H}_{2}$ and $\mathrm{C}_{2} \mathrm{H}_{6}$ bands); (b) fitting residual at $600-850 \mathrm{~cm}^{-1}$ region; (c) spectra at 1,225$1,325 \mathrm{~cm}^{-1}$ region $\left(\mathrm{CH}_{4}\right.$ bands); (d) fitting residual at 1,225-1,325 $\mathrm{cm}^{-1}$ region. CIRS observations are shown as black circles. The red, blue, orange, green and brown colours represent NEMESIS retrieval cases $\mathrm{C} 1-\mathrm{C} 5$, respectively. The goodness of fit $\left(\chi^{2} / N\right.$ where $N$ is the number of measurements) in the $600-850 \mathrm{~cm}^{-1}$ region is $\sim 0.5-0.6$ for each case. In the $\mathrm{CH}_{4}$ band, the goodness of fit is around unity for each case except for the brown case $\left(\chi^{2} / N=1.96\right)$, which does not fit the CIRS spectra. See Table 2 for detailed information of the cases.

until the CIRS spectra cannot be fitted within the measurement uncertainties. Table 2 summarized five typical tests. For each choice of optical constants, we performed an atmospheric retrieval of the Cassini CIRS spectra using NEMESIS model to constrain the aerosol opacity. Figure 5 illustrates the retrieval fitting results and residual values at $57^{\circ} \mathrm{S}$ and the values of goodness of fit are shown in Table 2 . The retrieved temperature profiles are shown in Fig. 6a. If we enhance the optical constants
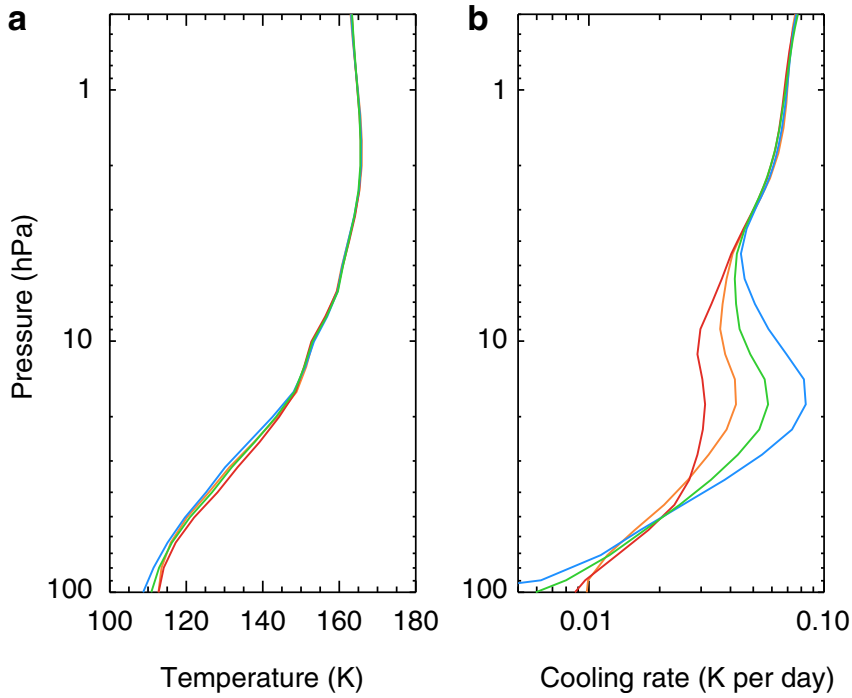

Figure 6 | Vertical temperature and cooling rate profiles at $57^{\circ} \mathrm{S}$.

(a) Retrieved temperature profiles; (b) corresponding zonally averaged cooling rates. The red, blue, orange and green colours represent cases $\mathrm{C} 1-\mathrm{C} 4$, respectively. The $\mathrm{C} 5$ case is not used because it cannot explain the CIRS observations.

from ref. 25 by a factor of 5 (case C5), we are not able to fit the $\mathrm{CH}_{4}$ emission spectra (Fig. 5). Overall, we estimated the upper limit of the aerosol optical depth in the mid-infrared wavelengths to be $\sim 0.1$ at $100 \mathrm{hPa}$ at high latitudes. This places an upper limit on the aerosol contribution to the globally averaged cooling flux of $\sim 0.2 \mathrm{~W} \mathrm{~m}^{-2}$ in the stratosphere (Fig. 1).

The aerosol cooling effect could be significant at middle and high latitudes where the particles are abundant but negligible at low latitudes. When the aerosol opacity is included, the cooling rate increases in the aerosol layer (Fig. 6b). At $57^{\circ} \mathrm{S}$, with a moderate choice of $k$ (case $\mathrm{C} 4$ ), the zonally averaged cooling rate including aerosols is $\sim 0.06 \mathrm{~K}$ per day. This is about two times larger than the gas-only cooling rate (case $\mathrm{C} 1$ ) at the pressures where the aerosol mixing ratio peaks $(\sim 10-20 \mathrm{hPa}$, Fig. $6 \mathrm{~b})$. The globally averaged aerosol cooling rate could be comparable to the gas cooling rate at $20 \mathrm{hPa}$ and partially compensate for the aerosol heating effect (Fig. 4f). On the other hand, the cooling rates from the aerosol cases are smaller than the pure-gas case at pressure levels below the aerosol layer (Fig. 6b), a result of energy conservation as constrained by the total emission observed by CIRS. Indeed, stronger aerosol absorption leads to a colder retrieved temperature profile (Fig. 6a). For instance, the temperature profile at $57^{\circ} \mathrm{S}$ from the $\mathrm{C} 2$ case is $\sim 3 \mathrm{~K}$ colder than the case without aerosols ( $\mathrm{Cl}$ case) below $\sim 40 \mathrm{hPa}$. This colder temperature leads to a smaller cooling effect than the pure-gas case $(\mathrm{C} 1)$ at pressures $>\sim 40 \mathrm{hPa}$ where the cooling is dominated by $\mathrm{H}_{2}-\mathrm{H}_{2}$ and $\mathrm{H}_{2}-\mathrm{He}$ CIA (Fig. 6b). 
We estimate the possible aerosol cooling rate for each latitude and their influences on the globally averaged cooling rate by combining the 'ensemble uncertainty' 4 of the temperature and gas abundances and the aerosol cooling rate tests. The uncertainty range including the aerosol contribution (Fig. 4f) is larger than that without aerosols (Fig. 4d) because the aerosols remain elusive from the CIRS spectra. Global radiative equilibrium is achievable when both aerosol heating and cooling are included (Fig. 4f).

\section{Discussion}

Owing to the lack of sufficient observational evidence before, the importance of aerosol heating in the middle atmosphere of Jupiter has long been a controversial question. It was suggested since the 1970s that the Jovian aerosols might absorb solar radiation and heat the atmosphere ${ }^{6-8}$. In the 1990s, based on the International Ultraviolet Explorer and Voyager-2 data, aerosol heating was shown to have a large impact on the atmospheric circulation $^{29}$. However, a later analysis using Hubble Space Telescope (HST) images found that the aerosols have relatively insignificant effects ${ }^{19}$. With better constraints on the spatial distribution and optical properties of aerosols, Cassini observations confirm a pronounced aerosol heating at high latitudes on Jupiter. Aside from the aerosol cooling effect, our conclusion is qualitatively consistent with previous estimate from the International Ultraviolet Explorer and Voyager- 2 data ${ }^{29}$ but with a much better spatial coverage. However, this work disagrees with heating rates derived solely on the basis of HST data ${ }^{3,19}$.

The major difference between the current work and previous studies is probably attributable to the fractal nature of the aerosol and aerosol spatial distribution. Through the multi-channelmulti-phase retrieval on Cassini images, we can characterize the fractal aggregates in great detail, including the optical depth, single scattering albedo and phase function of the particles. On the basis of low-phase-angle images alone ${ }^{19}$, the polar aerosols were assumed as tiny spherical particles of $\sim 0.07 \mu \mathrm{m}$ in radius instead of the submicron size aggregate particles. The latter have lower single scattering albedo than the spherical particles. Furthermore, with a larger particle size, the fractal aggregates have less backscattering than the spherical particles. Owing to the above two reasons, a larger total optical depth of the fractal aggregates is required to explain the low-phase-angle I/F observations, leading to a larger heating rate in this study, than that in ref. 19, at the south pole. Note that the $k$ values used in ref. 19 are lower than our nominal model but still within the sensitivity test range in our study.

The vertical distributions of aerosols in previous studies ${ }^{19,29}$ are based on microphysical simulations that are inconsistent with the near-infrared observations ${ }^{21}$. Previous studies adopted a haze layer located above $10 \mathrm{hPa}$ at polar regions, while the nearinfrared spectra reveal a main haze layer at $10-20 \mathrm{hPa}$ (ref. 20). West et al. ${ }^{29}$ only sampled two latitudes and estimated the other latitude information by scaling. The HST images ${ }^{19}$ have a good latitudinal coverage, but the aerosol heating appears influential only at the south pole, not at middle latitudes or at the north pole. Moreno et al. ${ }^{19}$ reported the aerosol optical depth at the north pole one order of magnitude less than that at the south pole. This result is inconsistent with other observations. For example, recent high-resolution ground-based near-infrared spectra ${ }^{22}$ concluded that the near-infrared haze optical depth at the northern pole is comparable to that at the southern pole. The Cassini images in low and high phase angles also revealed that the haze ultraviolet optical depth at north high latitudes is not significantly less than its south counterpart ${ }^{15}$. Furthermore, Cassini images show that the haze optical depth in the ultraviolet channel can approach unity down to $100 \mathrm{hPa}$ at middle latitudes ${ }^{15}$. Including the haze contribution at those latitudes would greatly enhance the aerosol heating. This will not only influence the local heat balance but also on the global energy equilibrium, especially at $10-20 \mathrm{hPa}$, as shown in our study.

Several other factors might also attribute to the difference between the current work and previous studies. For instance, we have a much better global coverage of the temperature, hydrocarbons and aerosols based on Cassini observations. The cooling rate in ref. 3 was likely to be underestimated because the temperature profile from Galileo entry probe is shown to be colder than the globally averaged temperature profile from Voyager and Cassini observations ${ }^{4}$, albeit the cooling rate in ref. 3 is still slightly larger than the gas heating rate at pressures $>10 \mathrm{hPa}$. The spectroscopic data of $\mathrm{CH}_{4}, \mathrm{C}_{2} \mathrm{H}_{2}$ and $\mathrm{C}_{2} \mathrm{H}_{6}$ have been greatly improved in the last decade (see Methods section for gas opacity). The state-of-the-art line data allow us to adopt the line-by-line approach to resolve the vibrational-rotational line shape of hydrocarbons ${ }^{4}$, the most accurate radiative transfer method to estimate the gas heating and cooling rate.

Another possible heating mechanism in the middle atmosphere is energy dissipation of upward propagating gravity waves from the troposphere. However, as per previous studies ${ }^{30,31}$, this hypothesis has several defects. First, there is little evidence of stratospheric gravity waves at middle and high latitudes. Second, there is no direct evidence of wave breaking in the lower stratosphere of Jupiter. Third, gravity wave breaking could either heat or cool the middle atmosphere but the net effect is difficult to quantify ${ }^{30}$.

Unlike the Earth, on which the photochemical product (ozone) only dominates the atmospheric radiative heating, Jupiter might exhibit a different regime of atmospheric energy balance where both the heating and cooling are significantly controlled by the photochemistry and auroral chemistry via the production of aerosols and $\mathrm{C}_{2}$ hydrocarbons. Aside from the first-order global energy balance, aerosol heating and cooling on Jupiter also influence the spatial distributions of radiative forcing, which has a significant impact on the large-scale dynamical circulation in the middle atmosphere ${ }^{5,19,29}$. The NASA JUNO spacecraft, arriving at Jupiter in 2016, will provide more insights on the aurora processes and aerosol formation in the polar region.

Jupiter is the second planetary body and the first hydrogendominated planet that shows evidence of hydrocarbon aerosols playing a significant role in regulating the radiation flux and most probably the circulation of its middle atmosphere. The other one is $\operatorname{Titan}^{32}$. Although Jupiter's atmosphere is primarily dominated by hydrogen, Titan's is dominated by nitrogen, both of these atmospheres produce fluffy, fractal aggregate particles, suggesting that fractal aggregates might be a ubiquitous result of hydrocarbon chemistry. In view of the existence of hydrocarbon aerosols in many other atmospheres dominated by hydrogen or nitrogen, such as those of Saturn ${ }^{33}$, Uranus ${ }^{34}$ and Neptune ${ }^{35}$, the early Earth $^{18}$, and possibly some exoplanets ${ }^{36-40}$, we hypothesize that the heating and cooling from fractal aggregates could also be important for determining the radiative energy distribution and climate evolution on these planets. Owing to their strong heating effects, fractal aggregates play a significant role in creating the temperature inversion in the lower stratosphere of Jupiter. They might also be partially responsible for the temperature inversions observed on the other giant planets in the Solar System, but neglected in previous studies ${ }^{41}$. A typical feature of the fluffy aggregate particle is its extremely low density, which might help to explain the existence of very high and thick haze layers at pressures $<0.1 \mathrm{hPa}$, as seen on the Neptune and sub-Neptune size planets GJ436b (ref. 39) and GJ1214b (ref. 40). A thorough study of fractal aggregates will shed light on how to characterize these particles in future photometry and polarization observations. 


\section{Methods}

Radiative heating and cooling model. For heating rate calculations between 0.20 and $0.94 \mu \mathrm{m}$ where the aerosol contribution is significant, we use a multiple scattering model based on the $\mathrm{C}$ version of the discrete ordinates radiative transfer code (DISORT Program for a multi-layered plane-parallel medium $)^{42}$. The phase function and cross sections of low-latitude particles were calculated based on Mie theory, while that of the fractal aggregates at middle and high latitudes were computed using a parameterization method for the aggregates with a fractal dimension of two ${ }^{17}$. The parameterization is based on electromagnetic scattering computations using the multi-sphere method ${ }^{43}$. In the heating rate calculations, we use 32 streams to characterize the intensity angular distribution, which displays almost no difference from the 64-stream case. A Gaussian quadrature method with 10 zenith angles is used to average the heating rates longitudinally. The spectral resolution is $0.001 \mu \mathrm{m}$. The effective cloud albedo in the troposphere is interpolated between 0.20 and $0.94 \mu \mathrm{m}$ based on the retrieved albedo from the UV1 channel and CB3/MT3 channels of Cassini ISS ${ }^{15}$

At longer wavelengths $(0.94-200 \mu \mathrm{m})$, our calculations adopt the line-by-line approach, based on a state-of-the-art high-resolution radiative heating and cooling model for the stratosphere of Jupiter, which has been rigorously validated against simple but realistic analytical solutions ${ }^{4}$. The $\mathrm{CH}_{4}$ heating rate calculation from 0.94 to $10 \mu \mathrm{m}$ is performed with a spectral resolution of $0.005 \mathrm{~cm}^{-1}$ to resolve the $\mathrm{CH}_{4}$ spectral line shape using the most current $\mathrm{CH}_{4}$ line lists (see discussion for gas opacity below). The thermal cooling rate from 50 to $2,500 \mathrm{~cm}^{-1}(4-200 \mu \mathrm{m})$ is calculated with a spectral resolution of $0.001 \mathrm{~cm}^{-1}$.

We calculate heating and cooling rates for every latitude to produce the latitudepressure two-dimensional maps. The specific heat of Jupiter's atmosphere is taken as $1.0998 \times 10^{4} \mathrm{~J} \mathrm{~kg}^{-1} \mathrm{~K}^{-1}$ (ref. 44 ). The globally averaged profiles are obtained via an area-weighted mean from $90^{\circ} \mathrm{N}$ to $90^{\circ} \mathrm{S}$. Since the data quality at latitudes north of $70^{\circ} \mathrm{N}$ and south of $70^{\circ} \mathrm{S}$ is not sufficiently good for rigorous atmospheric retrieval, we do not derive the atmospheric characteristics from the ISS images and CIRS spectra. Instead, we assume that the vertical profiles of aerosol, temperature and gases are identical to their values at $70^{\circ}$ north and south, respectively.

This assumption might introduce some uncertainty in our estimate of the global energy balance because the heating and cooling in the polar region, especially in the infrared aurora region, are not negligible. The polar aurora is known to be highly variable both temporally and spatially ${ }^{1}$. According to our limited data on the polar regions from Galileo ${ }^{45}$ and Cassini ${ }^{46}$ spacecraft as well as ground-based observations ${ }^{47,48}$, both regions poleward of $70^{\circ} \mathrm{N}$ and $70^{\circ} \mathrm{S}$ could be different from that $\sim 65^{\circ}-70^{\circ}$. For example, the Cassini CIRS instrument ${ }^{46}$ detected a variation of thermal emission over the $\mathrm{C}_{2} \mathrm{H}_{2}$ and $\mathrm{C}_{2} \mathrm{H}_{6}$ bands at regions poleward of $65^{\circ}$, within about a factor of 2 . However, because the surface area poleward of $70^{\circ}$ amounts to merely $6 \%$ of the total surface area of Jupiter, increasing the polar cooling rate by a factor of 2 will probably introduce an uncertainty of only $\sim 6 \%$ of the total cooling rate, which is still well-located within our estimated uncertainty range (Fig. 4f). On the other hand, the pressure level of infrared aurora source has not been precisely determined. If the emission originates from the upper stratosphere (for example, above $0.1 \mathrm{mbar}$ level), the aurora and its variability might have little impact on the thermal cooling rate at pressures we focus here. We should also point out that the aurora might also be associated with some heating mechanisms in the polar region that have not been considered in this study. Future analysis of the Jovian polar region will provide more information on the local energetics.

The aerosol and gas opacities used in the radiative calculations are discussed below.

Aerosol opacity sources. In the visible and near-infrared wavelengths, the early laboratory studies ${ }^{24-26}$ measured the optical properties of Titan-analogue aerosols (that is, in the nitrogen environment) and Jupiter-analogue aerosols (that is, in the hydrogen environment), respectively. Using the radio frequency glow discharge in $\mathrm{a} \mathrm{CH}_{4} / \mathrm{H}_{2}$ gas mixture, it was found that the refractive indices of these aerosols are consistent with high-phase-angle photometry data of Uranus by Voyager-2 at $0.55 \mu \mathrm{m}$ (ref. 24). Compared with aerosols produced in the $\mathrm{CH}_{4} / \mathrm{N}_{2}$ mixture ${ }^{26}$, the imaginary part of the refractive index of the Jupiter-analogue aerosols could be either larger or smaller, depending on the chemical composition. Unfortunately, ref. 24 has been the only laboratory experiment of the $\mathrm{CH}_{4} / \mathrm{H}_{2}$ gas mixture to date. It should be noted that the subsequent Titan-analogue laboratory measurements show that the aerosol properties are significantly influenced by the gas composition and environmental pressure ${ }^{25}$

Zhang et al..${ }^{15}$ combined the ground-based infrared spectra with the Cassini ISS observations at both low and high phase angles, and determined the $k$ values for the UV1 channel $(0.258 \mu \mathrm{m})$ and the infrared channels (CB3 at $0.938 \mu \mathrm{m}$ and MT3 at $0.889 \mu \mathrm{m}$ ). Owing to the existence of degeneracy, the $k$ value at UV1 channel varies from 0.008 to 0.02 and that at $0.9 \mu \mathrm{m}$ varies from 0.0001 to 0.004 . Different choices of $k$ would imply different solutions to fit the ISS data, such as the radius of the monomers from 10 to $40 \mathrm{~nm}$, the number of monomers per aggregate particle from 100 to 1,000 , and the abundance of particles. In all possible solutions, the total aerosol opacity only changes by $\sim 30 \%$ among all solutions. The corresponding aerosol heating rate does not change significantly.

For the heating rate calculations, we choose the model parameters for the $k$ values from ref. 15 as our nominal case (H1 in Table 1), with a careful sensitivity study in the radiatiave heating calculation. The $k$ value is $\sim 0.02$ at $0.258 \mu \mathrm{m}$ and a

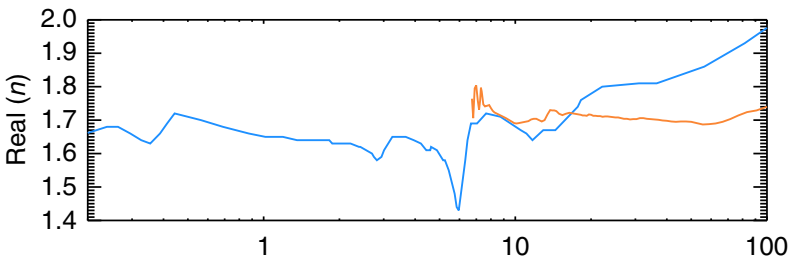

b

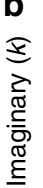

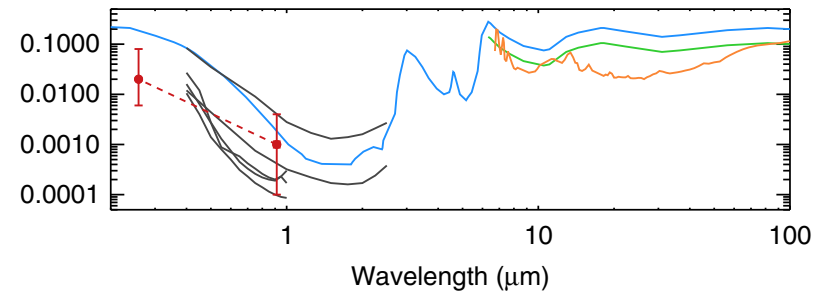

Figure 7 | Refractive indices of aerosols from 0.2 to $100 \mu \mathrm{m}$. (a) Real part of the refractive index; (b) imaginary part. The blue one is from the Titan tholin experiment ${ }^{26}$; orange is from Titan CIRS observations ${ }^{27}$; green is same as the blue one but reduced by a factor of 2 (case C4); black is the Jupiter-analogue aerosol experiment ${ }^{24}$; red is the derived from Cassini ISS observations ${ }^{15}$, with an interpolation in the coordinate of linear wavelength and logarithmic $k$ value. The red dashed line is used in the nominal case for the heating rate calculation (case $\mathrm{H} 1$ ) in this study.

0.001 at $\sim 0.9 \mu \mathrm{m}$. We performed an interpolation for the wavelengths between 0.20 and $0.94 \mu \mathrm{m}$ in the coordinate of linear wavelength and logarithmic $k$ value. The interpolation can be justified by the approximate same trend shown in the laboratory measurements (Fig. 7). As in ref. 15, we adopt the real part of refractive index $(n)$ from ref. 26.

Gas opacity sources. From 0.20 to $0.94 \mu \mathrm{m}$, the $\mathrm{CH}_{4}$ opacity is based on ref. 49 and Rayleigh scattering optical depth is taken from ref. 14. From 0.94 to $10 \mu \mathrm{m}$, a line-by-line calculation is performed. We compared several $\mathrm{CH}_{4}$ line databases, including the HITRAN2012 (refs 50,51), the database from ref. 22, and the M5 database ${ }^{52}$. For the $\mathrm{CH}_{4}$ broadening width, it has been suggested that line widths in the Jovian atmosphere $\left(\mathrm{H}_{2}-\mathrm{He}\right.$ mixture $)$ are similar to those in the Earth's atmosphere $\left(\mathrm{N}_{2}-\mathrm{O}_{2} \text { mixture }\right)^{22}$. All the above three $\mathrm{CH}_{4}$ opacity sources result in a consistent heating rate in our calculation. However, none of the above opacity sources covers the $\mathrm{CH}_{4}$ band between 0.94 and $1.1 \mu \mathrm{m}$. Recently, a '10 to 10 ' $\mathrm{CH}_{4}$ line database is computed from first principles ${ }^{53}$. This database is shown to be roughly consistent with the HITRAN2012 $\mathrm{CH}_{4}$ data in their overlapping nearinfrared wavelengths (personal communication with J. Bailey) and therefore is helpful to fill the gap between 0.94 and $1.1 \mu \mathrm{m}$ in our calculation. For this $\mathrm{CH}_{4}$ band, due to the lack of laboratory measurements of line shape parameters, we adopted an average pressure-broadened half-width of $0.06 \mathrm{~cm}^{-1}$ for foreignbroadening and $0.077 \mathrm{~cm}^{-1}$ for self-broadening at 1 bar pressure, and the temperature dependence exponent is $\sim 0.85$ (refs 52,54). The contribution from this band to the total heating rate is negligible (Fig. 2c). For the heating rate calculation, we obtain the near-infrared $\mathrm{H}_{2}-\mathrm{H}_{2}$ CIA absorption from refs 55,56 and $\mathrm{H}_{2}-\mathrm{He}$ CIA absorption from refs 57-60.

The thermal cooling rate is calculated from 50 to $2,500 \mathrm{~cm}^{-1}(4-200 \mu \mathrm{m})$. The opacity sources of $\mathrm{CH}_{4}, \mathrm{C}_{2} \mathrm{H}_{2}$ and $\mathrm{C}_{2} \mathrm{H}_{6}$ are obtained from HITRAN2012 with hydrogen-broadening widths $s^{4,61}$. Fractal aggregates are treated as pure absorbers in the thermal wavelengths due to their negligible single scattering albedo in the midinfrared. The mid-infrared $\mathrm{H}_{2}-\mathrm{H}_{2}$ and $\mathrm{H}_{2}-\mathrm{He}$ CIAs are obtained from ref. 62. Figure $2 \mathrm{c}$ shows the spectrally resolved heating/cooling rate as a function of wavelength and pressure, in which one can see the contributions from the CIAs and different vibrational-rotational bands from gases in Fig. $2 \mathrm{~b}$.

Spectral inversion model. The temperature and hydrocarbon distributions are simultaneously retrieved from the Cassini CIRS spectra ${ }^{4,10-12}$ using the NEMESIS algorithm ${ }^{28}$. This inversion model has been used in previous studies involving CIRS data retrieval ${ }^{4,11,12}$. In this study we extended the previous retrieval framework to include aerosol absorption in the mid-infrared wavelengths. No scattering calculations were needed as the fractal aggregate particles are significant absorbers and have negligible single scattering albedos at these wavelengths.

\section{References}

1. Moses, J. I. et al. in Jupiter-The Planet, Satellites and Magnetosphere (eds Bagenal, F., Dowling, T. \& McKinnon, W.) 129-157 (Cambridge University Press, 2004). 
2. Hanel, R., Conrath, B., Herath, L., Kunde, V. \& Pirraglia, J. Albedo, internal heat, and energy balance of Jupiter: Preliminary results of the Voyager infrared investigation. J. Geophys. Res. 86, 8705-8712 (1981).

3. Yelle, R. V., Griffith, C. A. \& Young, L. A. Structure of the Jovian stratosphere at the Galileo probe entry site. Icarus 152, 331-346 (2001).

4. Zhang, X. et al. Radiative forcing of the stratosphere of Jupiter, part I: atmospheric cooling rates from Voyager to Cassini. Planet. Space Sci. 88, 3-25 (2013).

5. Conrath, B. J., Gierasch, P. J. \& Leroy, S. S. Temperature and circulation in the stratosphere of the outer planets. Icarus 83, 255-281 (1990).

6. Wallace, L., Prather, M. \& Belton, M. J. S. The thermal structure of the atmosphere of Jupiter. Astrophys. J. 193, 481-493 (1974).

7. Cess, R. \& Chen, S. The influence of ethane and acetylene upon the thermal structure of the Jovian atmosphere. Icarus 26, 444-450 (1975).

8. Appleby, J. F. \& Joseph, S. H. Radiative-convective equilibrium models of Jupiter and Saturn. Icarus 59, 336-366 (1984).

9. Li, L. et al. Emitted power of Jupiter based on Cassini CIRS and VIMS observations. J. Geophys. Res. Planets 117, E11 (2012).

10. Simon-Miller, A. A. et al. Jupiter's atmospheric temperatures: from Voyager IRIS to Cassini CIRS. Icarus 180, 98-112 (2006)

11. Nixon, C. A. et al. Meridional variations of $\mathrm{C}_{2} \mathrm{H}_{2}$ and $\mathrm{C}_{2} \mathrm{H}_{6}$ in Jupiter's atmosphere from Cassini CIRS infrared spectra. Icarus 188, 47-71 (2007).

12. Nixon, C. A. et al. Abundances of Jupiter's trace hydrocarbons from Voyager and Cassini. Planet. Space Sci. 58, 1667-1680 (2010).

13. Wong, A. S., Yung, Y. L. \& Friedson, A. J. Benzene and haze formation in the polar atmosphere of Jupiter. Geophys. Res. Lett. 30, 1-4 (2003).

14. West, R. A. et al. in Jupiter-The Planet, Satellites and Magnetosphere (eds Bagenal, F., Dowling, T. \& McKinnon, W.) 79-104 (Cambridge University Press, 2004).

15. Zhang, X., West, R. A., Banfield, D. \& Yung, Y. L. Stratospheric aerosols on Jupiter from Cassini observations. Icarus 226, 159-171 (2013).

16. West, R. A. \& Smith, P. H. Evidence for aggregate particles in the atmospheres of Titan and Jupiter. Icarus 90, 330-333 (1991).

17. Tomasko, M. G. et al. A model of Titan's aerosols based on measurements made inside the atmosphere. Planet. Space Sci. 56, 669-707 (2008).

18. Wolf, E. T. \& Toon, O. B. Fractal organic Hazes provided an ultraviolet shield for early Earth. Science 328, 1266-1268 (2010).

19. Moreno, F. \& Sedano, J. Radiative balance and dynamics in the stratosphere of Jupiter: results from a latitude-dependent aerosol heating model. Icarus 130, 36-48 (1997).

20. Starodubtseva, O. M., Akimov, L. A. \& Korokhin, V. V. Seasonal variations in the north-south asymmetry of polarized light of Jupiter. Icarus 157, 419-425 (2002).

21. Banfield, D., Conrath, B. J., Gierasch, P. J. \& Nicholson, P. D. Near-IR spectrophotometry of Jovian aerosols-meridional and vertical distributions. Icarus 134, 11-23 (1998).

22. Kedziora-Chudczer, L. \& Bailey, J. Modelling the near-IR spectra of Jupiter using line-by-line methods. Mon. Not. R. Astron. Soc. 414, 1483-1492 (2011).

23. Wong, M. H., Mahaffy, P. R., Atreya, S. K., Niemann, H. B. \& Owen, T. C. Updated Galileo probe mass spectrometer measurements of carbon, oxygen, nitrogen, and sulfur on Jupiter. Icarus 171, 153-170 (2004).

24. Khare, B. N., Sagan, C., Thompson, W., Arakawa, E. \& Votaw, P. Solid hydrocarbon aerosols produced in simulated Uranian and Neptunian stratospheres. J. Geophys. Res. 92, 15067-15082 (1987).

25. Imanaka, H. et al. Laboratory experiments of Titan tholin formed in cold plasma at various pressures: implications for nitrogen-containing polycyclic aromatic compounds in Titan haze. Icarus 168, 344-366 (2004).

26. Khare, B. N. et al. Optical constants of organic tholins produced in a simulated Titanian atmosphere: from soft X-ray to microwave frequencies. Icarus $\mathbf{6 0}$, 127-137 (1984)

27. Vinatier, S. et al. Optical constants of Titan's stratospheric aerosols in the $70-1,500 \mathrm{~cm}^{-1}$ range constrained by Cassini/CIRS observations. Icarus 219 , 5-12 (2012)

28. Irwin, P. G. J. et al. The NEMESIS planetary atmosphere radiative transfer and retrieval tool. J. Quant. Spectrosc. Radiat. Transfer 109, 1136-1150 (2008)

29. West, R. A., Friedson, A. J. \& Appleby, J. Jovian large-scale stratospheric circulation. Icarus 100, 245-259 (1992).

30. Young, L. A., Yelle, R. V., Young, R., Seiff, A. \& Kirk, D. B. Gravity waves in Jupiter's stratosphere, as measured by the Galileo ASI experiment. Icarus 173, 185-199 (2005)

31. Watkins, C. \& Cho, J. Y. K. The vertical structure of Jupiter's equatorial zonal wind above the cloud deck, derived using mesoscale gravity waves. Geophys. Res. Lett. 40, 472-476 (2013).

32. Tomasko, M. G. et al. A model of Titan's aerosols based on measurements made inside the atmosphere. Planet. Space Sci. 56, 669-707 (2008).

33. West, R. A., Baines, K. H., Karkoschka, E. \& Sánchez-Lavega, A. in Saturn from Cassini-Huygens (eds Dougherty, M. K., Esposito, L. W. \& Krimigis, S. S. M.) 161-179 (Springer, 2009).
34. Pollack, J. B. et al. Nature of stratospheric haze on Uranus: evidence for condensed hydrocarbons. J. Geophys. Res. 92, 15037-15065 (1987).

35. Baines, K. H. \& Hammel, H. B. Clouds, hazes and stratospheric methane abundance ratio in Neptune. Icarus 109, 20-39 (1994).

36. Sing, D. K. et al. Hubble Space Telescope transmission spectroscopy of the exoplanet HD 189733b: high-altitude atmospheric haze in the optical and nearultraviolet with STIS. Mon. Not. R. Astron. Soc. 416, 1443-1455 (2011).

37. Sing, D. K. et al. HST hot-Jupiter transmission spectral survey: evidence for aerosols and lack of TiO in the atmosphere of WASP-12b. Mon. Not. R. Astron Soc 436, 2956-2973 (2013).

38. Deming, D. et al. Infrared transmission spectroscopy of the exoplanets HD $209458 \mathrm{~b}$ and XO-1b using the wide field camera-3 on the Hubble Space Telescope. Astrophys. J. 774, 95 (2013).

39. Knutson, H. A., Benneke, B., Deming, D. \& Homeier, D. A featureless transmission spectrum for the Neptune-mass exoplanet GJ436b. Nature 505, 66-68 (2014).

40. Kreidberg, L. et al. Clouds in the atmosphere of the super-Earth exoplanet GJ1214b. Nature 505, 69-72 (2014).

41. Robinson, T. D. \& Catling, D. C. Common 0.1-bar tropopause in thick atmospheres set by pressure-dependent infrared transparency. Nat. Geosci. 7, 12-15 (2014)

42. Buras, R., Dowling, T. \& Emde, C. New secondary-scattering correction in DISORT with increased efficiency for forward scattering. J. Quant. Spectrosc. Radiat. Transfer 112, 2028-2034 (2011).

43. Mishchenko, M. I., Travis, L. D. \& Mackowski, D. W. T-matrix computations of light scattering by nonspherical particles: A review. J. Quant. Spectrosc. Radiat. Transfer 55, 535-575 (1996).

44. Irwin, P. G. J. Giant Planets of Our Solar System: Atmospheres, Composition, and Structure (Springer, 2009).

45. Rages, K., Beebe, R. \& Senske, D. Jovian stratospheric hazes: the high phase angle view from Galileo. Icarus 139, 211-226 (1999).

46. Kunde, V. G. et al. Jupiter's atmospheric composition from the Cassini thermal infrared spectroscopy experiment. Science 305, 1582-1586 (2004).

47. Kostiuk, T., Romani, P. N., Espenak, F., Livengood, T. A. \& Goldstein, J. J. Temperature and abundances in the Jovian auroral stratosphere, 2. Ethylene as a probe of the microbar region. J. Geophys. Res. Planets 98, 18823-18830 (1993).

48. Livengood, T. A., Kostiuk, T., Espenak, F. \& Goldstein, J. J. Temperature and abundances in the Jovian auroral stratosphere, 1. Ethane as a probe of the millibar region. J. Geophys. Res. Planets 98, 18813-18822 (1993).

49. Karkoschka, E. \& Tomasko, M. G. Methane absorption coefficients for the Jovian planets from laboratory, Huygens, and HST data. Icarus 205, 674-694 (2010).

50. Brown, L. R. et al. Methane line parameters in the HITRAN2012 database. J. Quant. Spectrosc. Radiat. Transfer 130, 201-219 (2013).

51. Rothman, L. S. et al. The HITRAN2012 molecular spectroscopic database. J. Quant. Spectrosc. Radiat. Transfer 130, 4-50 (2013).

52. Sromovsky, L. A., Fry, P. M., Boudon, V., Campargue, A. \& Nikitin, A. Comparison of line-by-line and band models of near-IR methane absorption applied to outer planet atmospheres. Icarus 218, 1-23 (2012).

53. Yurchenko, S. N., Tennyson, J., Bailey, J., Hollis, M. D. \& Tinetti, G. Spectrum of hot methane in astronomical objects using a comprehensive computed line list. Proc. Natl Acad. Sci. USA 111, 9379-9383 (2014).

54. Nikitin, A. V. et al. GOSAT-2009 methane spectral line list in the 5,550-6,236 $\mathrm{cm}^{-1}$ range. J. Quant. Spectrosc. Radiat. Transfer 111, 2211-2224 (2010)

55. Borysow, A. Collision-induced absorption coefficients of $\mathrm{H}_{2}$ pairs at temperatures from $60 \mathrm{~K}$ to 1,000 K. Astron. Astrophys. 390, 779-782 (2002).

56. Borysow, A., Trafton, L., Frommhold, L. \& Birnbaum, G. Modeling of pressureinduced far-infrared absorption spectra: Molecular hydrogen pairs. Astrophys. $J$. 296, 644-654 (1985).

57. Borysow, J., Frommhold, L. \& Birnbaum, G. Collison-induced rototranslational absorption spectra of $\mathrm{H}_{2}$-He pairs at temperatures from 40 to $3000 \mathrm{~K}$. Astrophys. J. 326, 509-515 (1988).

58. Borysow, A. \& Frommhold, L. Collision-induced infrared spectra of $\mathrm{H}_{2}-\mathrm{He}$ pairs at temperatures from 18 to 7,000 K. II-Overtone and hot bands. Astrophys. J. 341, 549-555 (1989).

59. Borysow, A., Frommhold, L. \& Moraldi, M. Collision-induced infrared spectra of $\mathrm{H}_{2}-\mathrm{He}$ pairs involving 0-1 vibrational transitions and temperatures from 18 to 7,000 K. Astrophys. J. 336, 495-503 (1989).

60. Borysow, A. New model of collision-induced infrared absorption spectra of $\mathrm{H}_{2}$-He pairs in the 2-2.5 $\mu \mathrm{m}$ range at temperatures from 20 to $300 \mathrm{~K}$ : An update. Icarus 96, 169-175 (1992).

61. Orton, G. S. et al. Semi-annual oscillations in Saturn's low-latitude stratospheric temperatures. Nature 453, 196-199 (2008).

62. Orton, G. S., Gustafsson, M., Burgdorf, M. \& Meadows, V. Revised ab initio models for $\mathrm{H}_{2}-\mathrm{H}_{2}$ collision-induced absorption at low temperatures. Icarus $\mathbf{1 8 9}$, 544-549 (2007). 


\section{Acknowledgements}

We thank E. Karkoschka, L. Brown, G. Orton, J. Bailey, T. Kostiuk, A. Showman and L. Li for useful discussions and comments. Special thanks to M. Gerstell, P. Gao, R. Hu, P. Kopparla, C. Li, M.C. Liang, S. Newman, R.L. Shia, M. Wong, X. Xi and Q. Zhang for proofreading the manuscript. The early phase of this research was supported by the Outer Planets Research program via NASA Grant JPL 1452240 to the California Institute of Technology. R.A.W. and C.A.N. are supported by the NASA Cassini project. P.G.J.I. acknowledges the support of the UK Science and Technology Facilities Council

\section{Author contributions}

X.Z. carried out the radiative modelling and CIRS spectral retrieval; R.A.W. provided the ISS data; C.A.N. provided the CIRS data; R.A.W. and Y.L.Y. helped with radiative modelling; P.G.J.I. and C.A.N. helped with spectral inversion modelling; all authors contributed to the paper writing.

\section{Additional information}

Competing financial interests: The authors declare no competing financial interests.

Reprints and permission information is available online at http://npg.nature.com/ reprintsandpermissions/

How to cite this article: Zhang, X. et al. Aerosol influence on energy balance of the middle atmosphere of Jupiter. Nat. Commun. 6:10231 doi: 10.1038/ncomms10231 (2015).

(c) (i)

This work is licensed under a Creative Commons Attribution 4.0 International License. The images or other third party material in this article are included in the article's Creative Commons license, unless indicated otherwise in the credit line; if the material is not included under the Creative Commons license, users will need to obtain permission from the license holder to reproduce the material. To view a copy of this license, visit http://creativecommons.org/licenses/by/4.0/ 